\title{
Effects of the growth pattern of medulloblastoma on short-term neurological impairments after surgery: results from the prospective multicenter HIT-SIOP PNET 4 study
}

\author{
Elizabeth Schepke, MD, ${ }^{1,2}$ Magnus Tisell, MD, PhD, ${ }^{3,4}$ Colin Kennedy, MBBS, MD, ${ }^{5}$ \\ Stephanie Puget, MD, PhD, ${ }^{6}$ Paolo Ferroli, MD, PhD, Mathilde Chevignard, MD, PhD, ${ }^{8-10}$ \\ François Doz, MD, MSc, ${ }^{11,12}$ Barry Pizer, MD, PhD, ${ }^{13}$ Stefan Rutkowski, MD, ${ }^{14}$ \\ Maura Massimino, MD, PhD, ${ }^{15}$ Aurora Navajas, MD, PhD, ${ }^{16}$ Edward Schwalbe, PhD, ${ }^{17,18}$ \\ Debbie Hicks, PhD, ${ }^{17}$ Steven C. Clifford, PhD, ${ }^{17}$ Torsten Pietsch, MD, ${ }^{19}$ and \\ Birgitta Lannering, MD, PhD, ${ }^{20}$ on behalf of the SIOP-E Brain Tumor Group
}

\begin{abstract}
'Sahlgrenska Cancer Center, Department of Laboratory Medicine, Institute of Biomedicine, Sahlgrenska Academy, University of Gothenburg; '2Department of Pediatrics, Sahlgrenska University Hospital, Gothenburg; ${ }^{3}$ Department of Neurosurgery, Sahlgrenska University Hospital and ${ }^{4}$ Institute of Neuroscience and Physiology, Department of Neuroscience, Sahlgrenska Academy, University of Gothenburg, Sweden; ${ }^{5}$ University of Southampton Faculty of Medicine and University Hospital Southampton National Health Service Foundation Trust, Southampton, United Kingdom; ${ }^{6}$ Département de Neurochirurgie

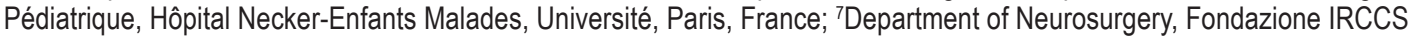
Istituto Neurologico Carlo Besta, Milan, Italy; ${ }^{8}$ Rehabilitation Department for Children with Acquired Neurological Injury, Saint Maurice Hospitals, Saint Maurice; ' 2 Laboratoire d'Imagerie Biomédicale and ${ }^{10} \mathrm{GRC} 24 \mathrm{HaMCRe}$, Sorbonne Université, Paris; ${ }^{11}$ SIREDO Cancer Center (Care, Innovation \& Research, in Childhood, Adolescent and Young-Adult Oncology), Institut Curie Paris; ${ }^{12}$ Department of Pediatrics, University Paris Descartes, Paris, France; ${ }^{13}$ Department of Oncology, Alder Hey Children's NHS Foundation Trust, Liverpool, United Kingdom; ${ }^{14}$ Department of Pediatric Hematology and Oncology, University Medical Center Hamburg-Eppendorf, Hamburg, Germany; ${ }^{15}$ Fondazione Istituto di Ricovero e Cura a Carattere Scientifico IRCCS Istituto Nazionale dei Tumori, Milan, Italy; ${ }^{16}$ BioCruces Health Research Institute, Bilbao, Spain; ${ }^{17 W o l f s o n ~ C h i l d h o o d ~ C a n c e r ~}$ Research Centre, Northern Institute for Cancer Research, Newcastle University, Newcastle-upon-Tyne; ${ }^{18}$ Department of Applied Sciences, Northumbria University, Newcastle-upon-Tyne, United Kingdom; ${ }^{19}$ Institute of Neuropathology, DGNN Brain Tumour Reference Center, University of Bonn, Germany; and ${ }^{20}$ Department of Pediatrics, Institute of Clinical Sciences, Sahlgrenska Academy, University of Gothenburg, Germany
\end{abstract}

OBJECTIVE Extensive resection of a tumor in the posterior fossa in children is associated with the risk of neurological deficits. The objective of this study was to prospectively evaluate the short-term neurological morbidity in children after medulloblastoma surgery and relate this to the tumor's growth pattern and to the extent of resection.

METHODS In 160 patients taking part in the HIT-SIOP PNET 4 (Hyperfractionated Versus Conventionally Fractionated Radiotherapy in Standard Risk Medulloblastoma) trial, neurosurgeons prospectively responded to questions concerning the growth pattern of the tumor they had resected. The extent of resection (gross, near, or subtotal) was evaluated using MRI. The patients' neurological status before resection and around 30 days after resection was recorded.

RESULTS Invasive tumor growth, defined as local invasion in the brain or meninges, cranial nerve, or major vessel, was reported in $58 \%$ of the patients. After surgery almost $70 \%$ of all patients were affected by one or several neurological impairments (e.g., impaired vision, impaired extraocular movements, and ataxia). However, this figure was very similar to the preoperative findings. Invasive tumor growth implied a significantly higher number of impairments after surgery ( $p$ $=0.03)$ and greater deterioration regarding extraocular movements $(p=0.012)$, facial weakness $(p=0.048)$, and ataxia in the arms $(p=0.014)$ and trunk $(p=0.025)$ compared with noninvasive tumor growth. This deterioration was not dependent on the extent of resection performed. Progression-free survival (PFS) at 5 years was $80 \% \pm 4 \%$ and $76 \% \pm 5 \%$ for patients with invasive and noninvasive tumor growth, respectively, with no difference in the 5-year PFS for extent of resection.

ABBREVIATIONS CMS = cerebellar mutism syndrome; GTR = gross-total resection; NTR = near-total resection; PFS = progression-free survival; STR = subtotal resection. SUBMITTED June 19, 2019. ACCEPTED November 1, 2019.

INCLUDE WHEN CITING Published online January 17, 2020; DOI: 10.3171/2019.11.PEDS19349. 
CONCLUSIONS Preoperative neurological impairments and invasive tumor growth were strong predictors of deterioration in short-term neurological outcome after medulloblastoma neurosurgery, whereas the extent of resection was not. Neither tumor invasiveness nor extent of resection influenced PFS. These findings support the continuation of maximal safe resection in medulloblastoma surgery where functional risks are not taken in areas with tumor invasion.

https://thejns.org/doi/abs/10.3171/2019.11.PEDS19349

KEYWORDS neurological sequelae; resection; growth pattern; medulloblastoma; pediatric neurosurgery; cerebellar mutism syndrome; CMS; oncology

M EDUlloblastoma is the most common embryonal brain tumor in children, representing almost $20 \%$ of all childhood primary CNS tumors. Medulloblastoma originates in the posterior cranial fossa, most often from the cerebellar vermis in the roof of the fourth ventricle. The revised WHO classification of CNS tumors (2016) defined 4 medulloblastoma disease entities (WNT, SHH-TP53 wildtype, SHH-TP53 mutant, and nonWNT/non-SHH) with distinct cellular origin, localization, histopathological characteristics, molecular genetic features, and clinical behavior. ${ }^{21,23,30,34}$ The prognosis of nondisseminated medulloblastoma is, in addition to the histopathology, dependent on the genetic features of the tumor as well as clinical factors. The treatment of medulloblastoma involves surgery followed by radiotherapy and chemotherapy. Today the 5-year overall survival for standard-risk medulloblastoma is expected to be around $80 \% .20$

The prognostic importance of gross-total resection (GTR) versus near-total resection (NTR) or subtotal resection (STR) for survival is unclear. ${ }^{5,11,12,35}$ Recent publications have put more emphasis on the prognostic importance of the genetically defined entity of the tumor for survival ${ }^{24,29}$ and no benefit of GTR compared with NTR or STR on survival..$^{35,36}$

Concerning morbidity, extensive resection of a tumor in the posterior fossa may be associated with increased risk for neurological deficits, both short- and long-term. ${ }^{32,37}$ Children with medulloblastoma have been reported to encounter mutism, new cranial nerve palsies, and worsening of ataxia after surgery in $24 \%-44 \%$ of cases with increasing incidence when undergoing GTR.,10 Extensive resection of medulloblastomas invasive into the brainstem causes a considerable neurological risk and no benefit in progression-free survival (PFS).$^{10}$ Cerebellar mutism syndrome (CMS), characterized by mutism and emotional lability, ${ }^{14,25}$ is a negative prognostic factor for short- and long-term neurological outcome. ${ }^{17,33}$ Although its pathogenesis is most likely multifactorial, ${ }^{3,8}$ it might be increased after extensive resection compared with less complete resections. ${ }^{19}$

When neurosurgeons perform tumor resection, the final neuropathological diagnosis is not known, and the accomplished extent of resection is highly dependent on the growth pattern of the tumor as well as on the experience and skills of the surgeon. In the literature, there are few reports on the impact of the growth pattern of medulloblastoma on neurological morbidity after surgery.

The aim of the present study was to investigate the short-term neurological deficits in children who underwent surgery for medulloblastoma and relate this to the growth pattern and the extent of resection as well as to PFS. This information was prospectively collected in the multicenter HIT-SIOP PNET 4 (Hyperfractionated Versus Conventionally Fractionated Radiotherapy in Standard Risk Medulloblastoma) study.

\section{Methods}

The international phase 3 multicenter study HIT-SIOP PNET 4 recruited 338 patients with standard-risk medulloblastoma from 10 European countries between 2001 and 2006 (clinical trial registration no. NCT01351870, clinicaltrials.gov). ${ }^{20}$ The patients were $4-21$ years of age at inclusion. Standard-risk medulloblastoma was defined as no sign of metastatic disease on preoperative MRI or cerebrospinal fluid cytology. Postoperative residual tumors of any size were allowed. At diagnosis, tumor material was centrally reviewed by experienced neuropathologists, confirming the diagnosis and the histological medulloblastoma entity. Epigenetic subgroup analysis and cytogenetic evaluation were performed later but only in a fraction of the cohort. ${ }^{11}$ The randomized question concerned the use of conventional or hyperfractionated radiotherapy and showed no difference in survival rate. ${ }^{20}$

The treatment protocol included a prospective collection of neurosurgical data with pre- and postoperative neurological status evaluation. Seventy different neurosurgical centers in 9 European countries took part in this substudy. The study was approved by each national/institutional review board, and informed consent was obtained from all patients, parents, and/or guardians.

After surgery, the neurosurgeons filled out a questionnaire concerning the growth pattern of the tumor regarding the presence of local brain or leptomeningeal invasion or involvement of a cranial nerve or major vessels. Thus, invasive tumor growth was defined by the neurosurgeon as the presence of any of these factors. The neurosurgeons were also asked about the techniques employed (e.g., use of an ultrasonic aspirator or operating microscope and management of hydrocephalus). The hydrocephalus issue included yes or no answers to the use of preoperative steroids, pre- or intraoperative external ventricular drain, ventricular shunt or puncture, or neuroendoscopic third ventriculostomy. No information about the surgical approach was collected. Intraoperative limiting factors such as blood loss, brain swelling, cardiovascular changes, inadequate access, and premature halting of the operation 
were reported as a yes or no answer. Operation time was recorded.

Data collected on preoperative MRI included the tumor size but not the growth pattern of the tumor or the more precise localization of the tumor. Postoperative MRI was performed, most often within 72 hours postsurgery in all patients but one, who underwent CT scanning instead. The images were centrally reviewed in each participating country, and the extent of resection was reported. GTR was defined as there being no sign of residual tumor, NTR as a residual tumor less than or equal to $1.5 \mathrm{~cm}^{2}$ (largest diameter in the axial plane according to protocol), and STR as a residual tumor more than $1.5 \mathrm{~cm}^{2} .{ }^{20}$

The following general complications within 30 days after surgery were recorded: seizures, CMS, intracranial hemorrhage, hydrocephalus requiring operation, CNS infection, other infection, subcutaneous CSF collection, and readmission to intensive care. These complications were reported by a yes or no answer on the questionnaire. Time to regain full consciousness was also recorded.

Neurological status before and around 30 days after surgery (before the start of radiotherapy) was recorded by a physician. The neurological record stated whether the patient had full or impaired consciousness, whether vision was impaired, and whether the patient had impairment of extraocular movements or palate or tongue movements. The presence of facial weakness, ataxia of the arms and trunk, and limb weakness was also recorded. In this present study, 2 ways of studying neurological deficits were used. First, the number of impairments for each patient was added and compared before and after surgery; and second, to analyze which neurological functions were most likely to be affected by surgery, we compared each patient's neurological deficits before and after tumor resection and graded the change in each neurological impairment as better, same, or worse.

Patient follow-up is part of the HIT-SIOP PNET 4 study. Events are continuously registered in the database by each national study group.

\section{Methylation Analysis}

Epigenetic subgrouping of the tumors was performed in all patients with sufficient remaining tumor material left $(\mathrm{n}=74)$, as previously described. ${ }^{11,28}$

\section{Statistical Analysis}

Kaplan-Meier survival plots and logistic regression were performed in $\mathrm{R}$ using the survival and stats packages. For all other statistical analyses, IBM SPSS (version 25, IBM Corp.) was used. Comparisons of categorical variables between groups were performed using McNemar's test, chi-square test, and Fisher's exact test where appropriate. Prognostic factors for neurological deficits after surgery were evaluated using logistic regression analysis, with sex, age, tumor size, tumor growth pattern, extent of resection, and a categorical variable denoting whether there were fewer than 2 or 2 or more deficits before surgery as covariates. Complete data for the logistic regression analysis was available for 120 patients. The PFS was estimated using the Kaplan-Meier method, and differenc- es in outcome between patient groups were tested using the log-rank method; $\mathrm{p}<0.05$ was considered significant.

\section{Results \\ Patients}

A total of 160 eligible patients with standard-risk medulloblastoma and neurosurgical data were identified. Patient characteristics are listed in Table 1. There were 99 male and 61 female patients with a sex ratio of 1.6. The mean age at diagnosis was 9.4 years (range 3.2-19.3 years). The tumor size was between 1.9 and $9.5 \mathrm{~cm}$ in its largest diameter (mean $4.3 \mathrm{~cm}$ ). The mean operation time was 4 hours, 30 minutes (range 1 hour, 40 minutes to 8 hours, 30 minutes). The operation techniques used were very similar in all centers; for example, 95\% used an operating microscope and $80 \%$ an ultrasonic aspirator. Very few intraoperative limiting factors were reported (data not shown).

The vast majority of centers reported 1-2 patients to the study. Eight centers reported 5-13 patients, each who together composed one-third of the study population.

\section{Tumor Growth and Resection}

The surgeon's description of the tumor's growth pattern was available for 158 patients. It was reported as invasive in 91 patients $(58 \%)$ and noninvasive in 67 patients $(42 \%)$ (Table 1). The majority of invasive tumors (in 72 of 91 patients) had local brain invasion reported as the only sign of invasive growth. Nineteen patients had a combination of growth in a major vessel or cranial nerve or local invasiveness (local brain or leptomeningeal) reported. The 5 most invasive tumors exhibited local brain invasion as well as growth in a cranial nerve and involvement of a major vessel.

A GTR was performed in $129(80 \%)$ patients, an NTR in $25(16 \%)$, and an STR in $6(4 \%)$. As shown in Table 1 , the extent of resection was similarly distributed in invasive and noninvasive tumors. Eight patients underwent secondary surgery due to a residual tumor, and postoperative MRI thereafter showed GTR in 6 patients and NTR in 2 .

\section{General Complications Within 30 Days Postresection}

General complications were reported in $19 \%$ of the patients (Table 2). The time to full recovery of consciousness after surgery showed great variation ( $0-48$ days); however, $75 \%$ of patients had fully recovered within 1 day. The patient with the longest recovery time, 48 days, had CMS and is a long-term survivor.

\section{Neurological Deficits as a Result of Tumor Growth Pattern and Resection}

Both pre- and postresection, $68 \%$ and $69 \%$ of patients were affected by at least 1 neurological impairment, respectively. Thus, $30 \%$ of the patients had no neurological impairment either before or after surgery. The frequency of the most common preoperative impairments, such as vision impairment and ataxia of arms and trunk, remained essentially unchanged after surgery (Table 3). Improve- 
TABLE 1. Clinical and tumor characteristics in 160 children treated for medulloblastoma in the HIT-SIOP PNET 4 study

\begin{tabular}{|c|c|}
\hline & Value \\
\hline Male & $99(62)$ \\
\hline Female & $61(38)$ \\
\hline Mean age at diagnosis, yrs (range) & $9.4(3.2-19.3)$ \\
\hline Mean tumor size, $\mathrm{cm}$ (range) & $4.3(1.9-9.5)$ \\
\hline Operation time, range & $1 \mathrm{hr}, 40$ mins to $8 \mathrm{hrs}, 30 \mathrm{mins}$ \\
\hline \multicolumn{2}{|l|}{ Extent of resection* } \\
\hline GTR & $129(80)$ \\
\hline NTR ( $\leq 1.5-\mathrm{cm}^{2}$ residual tumor) & $25(16)$ \\
\hline STR (>1.5- $\mathrm{cm}^{2}$ residual tumor) & $6(4)$ \\
\hline \multicolumn{2}{|l|}{ Growth pattern† } \\
\hline Invasive signs ( $\geq 1$ per patient) & $91(58)$ \\
\hline Local brain invasion & $87(55)$ \\
\hline Leptomeningeal invasion & $7(4.4)$ \\
\hline Major vessel involvement & $15(9.5)$ \\
\hline $\mathrm{CN}$ invasion & $9(6)$ \\
\hline Noninvasive & $67(42)$ \\
\hline \multicolumn{2}{|l|}{ Growth pattern \& extent of resection } \\
\hline \multicolumn{2}{|l|}{ Invasive growth } \\
\hline GTR & $73(80)$ \\
\hline NTR & $14(15)$ \\
\hline STR & $4(4)$ \\
\hline \multicolumn{2}{|l|}{ Noninvasive growth } \\
\hline GTR & $54(81)$ \\
\hline NTR & $11(16)$ \\
\hline STR & $2(3)$ \\
\hline Preop steroids & $115(77)$ \\
\hline Molecular subgroups & $74(46)$ \\
\hline WNT & $10(14)$ \\
\hline $\mathrm{SHH}$ & $16(22)$ \\
\hline Non-WNT/non-SHH & $48(65)$ \\
\hline \multicolumn{2}{|c|}{$\begin{array}{l}\mathrm{CN}=\text { cranial nerve. } \\
\text { Values are presented as the number of patients (\%) unless stated otherwise. } \\
{ }^{*} \text { As noted on postoperative radiology. } \\
\dagger \text { Based on the neurosurgical report. }\end{array}$} \\
\hline
\end{tabular}

ment after surgery was seen for impaired consciousness. Deterioration was seen mainly for extraocular movements $(\mathrm{p}=0.03)$, facial weakness $(\mathrm{p}<0.01)$, and limb weakness $(\mathrm{p}<0.01)$.

Half of all patients had no impairment or only a single neurological impairment before and after surgery, which in individual patients could mean deterioration or improvement (see below). The other half had 2 or more impairments. Before resection, there was no difference in the number of neurological deficits in the 91 patients with invasive tumor growth compared with those with noninvasive growth. After resection, there was a significantly higher percentage of patients with 2 or more impairments in the patients with invasive tumor growth compared with those with noninvasive growth $(57 \%$ and $37 \%$, respectively; $p=0.03$ ) (Table 4), giving an OR of 2.15. This differ-
TABLE 2. General complications within 30 days postresection

\begin{tabular}{lc}
\hline & Value \\
\hline CMS & $19(13)$ \\
\hline Subcutaneous CSF collection & $10(7)$ \\
\hline Readmission to intensive care & $9(6)$ \\
\hline CNS infection & $7(5)$ \\
\hline Other infection & $7(5)$ \\
\hline Hydrocephalus requiring operation postresection & $8(5)$ \\
\hline Seizures & $4(3)$ \\
\hline Intracranial hemorrhage & $4(3)$ \\
\hline Mean days btwn surgery \& full recovery of & $2.2(0-48)$ \\
\hline
\end{tabular}

Data are presented as number of patients (\%) unless stated otherwise.

ence in number of postoperative neurological impairments was not seen when comparing the extent of resection performed (Table 4).

Looking at specific neurological impairments in the 91 patients with an invasive tumor, the evolution of these was significantly worsened postresection compared with the patients in whom the tumor was noninvasive. This was true for extraocular movements $(\mathrm{p}=0.012)$, facial weakness $(\mathrm{p}=0.048)$, ataxia of the arms $(\mathrm{p}=0.014)$, and truncal ataxia $(\mathrm{p}=0.025)$ (Table 5). This worsening in neurological outcome after surgery was independent of the extent of surgical resection (data not shown).

A multivariate analysis was performed for all patients with complete data of the preoperative factors: age, sex, number of preoperative neurological impairments, tumor size, tumor growth pattern, and extent of resection. The number of postoperative impairments was positively associated with the number of preoperative impairments and the degree of tumor invasiveness $(\mathrm{p}<0.05$ for both, OR 7 and 3 , respectively) but not with the extent of resection ( $p$ $=0.5$ ). Every increase in centimeters of the tumor size also increased the risk of having more postoperative deficits, with an OR of 1.75 .

\section{Molecular Subgroup Analysis}

In 74 patients, tumor material was available for molecular subgroup analysis (Table 1). In the 24 tumors with subgroups WNT and SHH, invasive tumor growth was seen in 50\% and $43 \%$, respectively, and in non-WNT/non$\mathrm{SHH}$ in 58\%. In tumors with subgroup WNT and SHH, a GTR was performed in $90 \%-100 \%$ of patients, and in non-WNT/non-SHH it was performed in $77 \%$ of patients (not significant). There was no correlation between subgroup and number of impairments.

\section{Cerebellar Mutism Syndrome}

Cerebellar mutism syndrome was recorded to be present in $13 \%$ of the children. In patients with invasive tumor $13 \%$ developed CMS and in patients with noninvasive tumor growth $10 \%$. CMS was only present in patients who underwent GTR or NTR but not in the 6 patients with STR. 
TABLE 3. Occurrence of pre- and postoperative neurological impairments within 30 days postresection

\begin{tabular}{lcccc}
\hline \multicolumn{1}{c}{ Neurological Impairment } & $\begin{array}{c}\text { Preresection, } \\
\%\end{array}$ & $\begin{array}{c}\text { Postresection, } \\
\%\end{array}$ & $\mathrm{p} \mathrm{Value}{ }^{*}$ & $95 \% \mathrm{Cl}$ \\
\hline Impaired consciousness $(\mathrm{n}=148)$ & 8 & 2 & 0.02 & 0.01 to 0.1 \\
\hline Impaired vision for age $(\mathrm{n}=145)$ & 24 & 25 & $>0.99$ & \\
\hline Impaired extraocular movements $(\mathrm{n}=143)$ & 25 & 36 & 0.03 & -0.2 to -0.02 \\
\hline Facial weakness $(\mathrm{n}=141)$ & 3 & 10 & $<0.01$ & -0.1 to -0.03 \\
\hline Impaired palate/tongue movements $(\mathrm{n}=140)$ & $<1$ & 4 & 0.2 & \\
\hline Limb weakness $(\mathrm{n}=140)$ & 10 & 26 & $<0.01$ & -0.2 to -0.09 \\
\hline Ataxia arms $(\mathrm{n}=136)$ & 37 & 46 & 0.09 & \\
\hline Ataxia trunk $(\mathrm{n}=135)$ & 39 & 39 & 0.86 & \\
\hline
\end{tabular}

* McNemar's chi-square test; statistical analysis excluded nonpaired data.

\section{Management of Hydrocephalus}

Seventy-seven percent of all patients received preoperative steroids. Of the 153 patients for which we had information about the management of hydrocephalus, 74 (48\%) required an operation for hydrocephalus (ventriculostomy, ventricular shunt, or external drainage preoperatively or intraoperatively).

\section{Long-Term PFS}

At 5 years from diagnosis, PFS was $80 \% \pm 4 \%$ and $76 \% \pm 5 \%$ for patients with invasive and noninvasive tumor growth, respectively, with no significant difference between the groups ( $\mathrm{p}=0.8$ ) (Fig. 1). The 5-year PFS was $77 \% \pm 4 \%$ for GTR, $88 \% \pm 7 \%$ for NTR, and $83 \% \pm 15 \%$ for STR with no significant difference between the groups.

\section{Discussion}

When discussing the 3 cornerstones in the treatment for medulloblastoma (surgery, radiotherapy, and chemotherapy) and their propensities to cause morbidity, the least focus has been on surgery. Therefore, we studied neurological status before and after surgery related to neurosurgical conditions, which were prospectively collected in the international multicenter study HIT-SIOP PNET 4. Our main finding is that patients with an invasive tumor growth were more likely to experience deterioration in their neurological impairments between pre- and postoperative evaluations.

In this study, we report on short-term neurological findings after surgery. Many studies have shown that patients undergoing treatment for medulloblastoma, including neurosurgery, have long-term neurological and neurocognitive problems..$^{22,38}$ Most of these findings have been attributed to treatment with radiotherapy. In the HIT-SIOP PNET 4 study, younger children who underwent hyperfractionated compared with conventional radiotherapy had better executive function and marginally higher verbal IQ but without accompanying change in health status, behavior, or quality of life. ${ }^{6,18}$ Apart from studies looking at the long-term effects of CMS, ${ }^{17,33}$ there are only a few studies linking long-term neurological sequelae also to surgeryrelated factors. ${ }^{13}$ Naturally surgical short-term damage has bearing on the long-term neurological outcome.

To our knowledge, there have been no studies on the impact of tumor invasiveness on neurological morbidity. In this study, when comparing the number of neurological impairments pre- and postresection we found that patients with invasive tumor growth had a more than 2-fold-higher risk of having 2 or more neurological deficits after surgery compared with patients with noninvasive tumor growth. When looking at the changes (better, same, worse) in neurological symptoms postresection, patients with invasive tumor growth had a significant deterioration of sev-

TABLE 4. Neurological impairments postresection in patients with a tumor with an invasive or noninvasive growth pattern and GTR, NTR, or STR

\begin{tabular}{|c|c|c|c|c|}
\hline & \multicolumn{2}{|c|}{ No. of Neurological Impairments* } & \multirow[b]{2}{*}{$\mathrm{p}$ Value $†$} & \multirow[b]{2}{*}{$95 \% \mathrm{Cl}$} \\
\hline & 0 or 1 & $\geq 2$ & & \\
\hline Invasive tumor growth $(n=83)$ & 43 & 57 & \multirow{2}{*}{0.03} & \multirow{2}{*}{$0.02-0.37$} \\
\hline Noninvasive tumor growth $(n=62)$ & 63 & 37 & & \\
\hline $\operatorname{GTR}(n=120)$ & 52 & 48 & $>0.99$ & \\
\hline $\operatorname{NTR}(n=21)$ & 52 & 48 & & \\
\hline $\operatorname{STR}(n=6)$ & 50 & 50 & & \\
\hline
\end{tabular}


TABLE 5. Change in neurological impairment related to invasive tumor growth or noninvasive tumor growth

\begin{tabular}{|c|c|c|c|}
\hline & $\begin{array}{l}\text { Invasive Tumor Growth, } \\
\qquad \%(n=91)\end{array}$ & $\begin{array}{l}\text { Noninvasive Tumor Growth, } \\
\qquad \%(n=67)\end{array}$ & $p$ Value ${ }^{*}$ \\
\hline Vision for age & & & 0.523 \\
\hline Worse & 10.7 & 5.4 & \\
\hline Same & 82.7 & 85.7 & \\
\hline Better & 6.7 & 8.9 & \\
\hline CN palsy of III, IV, or VI & & & 0.012 \\
\hline Worse & 24.7 & 8.2 & \\
\hline Same & 65.4 & 86.9 & \\
\hline Better & 9.9 & 4.9 & \\
\hline CN palsy of IX, X, or XI & & & 0.076 \\
\hline Worse & 0 & 5.1 & \\
\hline Same & 100 & 94.6 & \\
\hline Better & 0 & 0 & \\
\hline Facial weakness & & & 0.048 \\
\hline Worse & 12.5 & 3.3 & \\
\hline Same & 87.5 & 95 & \\
\hline Better & 0 & 1.7 & \\
\hline Arm ataxia & & & 0.014 \\
\hline Worse & 23.4 & 6.9 & \\
\hline Same & 71.4 & 79.3 & \\
\hline Better & 5.2 & 13.8 & \\
\hline Truncal ataxia & & & 0.025 \\
\hline Worse & 20.3 & 5.3 & \\
\hline Same & 70.9 & 78.6 & \\
\hline Better & 8.9 & 15.8 & \\
\hline Limb weakness & & & 0.124 \\
\hline Worse & 23.5 & 11.7 & \\
\hline Same & 72.8 & 86.7 & \\
\hline Better & 3.7 & 1.7 & \\
\hline
\end{tabular}

eral neurological impairments postsurgery compared with noninvasive tumors. This deterioration was not dependent on the extent of resection performed. There was also no difference regarding the number of or worsening of neurological deficits if tumors with invasive growth were resected totally or near/subtotally. It thus seems that the invasiveness of the tumor growth per se could be important for the outcome of neurological deficits. This finding was confirmed in a multivariate analysis. Certainly, this result may suggest that the neurosurgeons made the appropriate decision concerning the extent of resection in the individual cases. Many medulloblastomas grow adherent to the floor of the fourth ventricle and to the brainstem where neurosurgeons have made decisions to resect with caution. This may have contributed to the finding of no clear relationship between extent of resection and postsurgical neurological status.

However, the patient's postoperative status was to the highest degree dependent on the preoperative status, which in turn was not different in invasive and noninvasive tumors. In fact, in our study, when looking at all patients, we found the same percentage of patients $(68 \%$ and $69 \%$, respectively) affected by at least 1 neurological impairment before and after surgery. This high percentage after short-term follow-up has also been noted in previous studies for posterior fossa tumors in general. ${ }^{16}$ However, despite this overall stable finding of pre- and postsurgery deficits, some patients improved and some deteriorated. Not surprisingly, ataxia of the trunk and arms as well as impaired vision and palsy of cranial nerves III, IV, and VI were the most common neurological deficits before and a short time after surgery.

Thus, it seems that for this cohort, surgery did not put the patients at risk for increased neurological morbidity. In individual patients exceptions occurred in both ways. An exception was CMS, which is never present before surgery. This study showed a relatively low frequency of CMS, ${ }^{15,26}$ which may be due to the fact that the definition of CMS was made by each center and could have been underreported in the absence of a standardized scale. Still, we did 


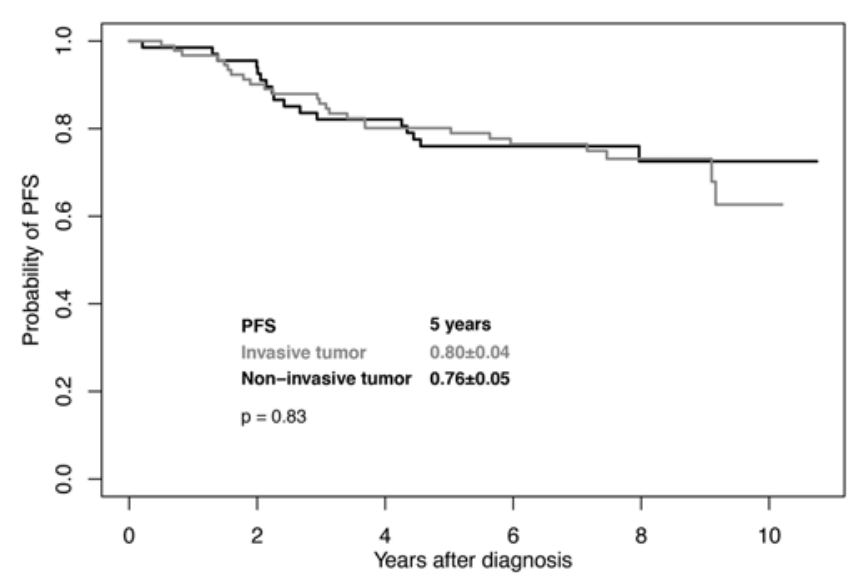

FIG. 1. Long-term probability of PFS (Kaplan-Meier, log-rank test) in relation to invasive or noninvasive medulloblastoma tumor growth, as defined by the neurosurgeon intraoperatively $(n=158)$.

not find that tumor invasiveness or extent of resection was related to an increased risk of CMS.

Tumor invasiveness did not affect cure in this study. Tumors with invasive tumor growth had a 5-year PFS of $80 \%$ $\pm 4 \%$, which is in line with previous publications from the HIT SIOP-PNET 4.20,27

The importance of resection for cure in patients with medulloblastomas is widely established. We also found, in a study of relapse in this patient cohort, that surgery for relapse was associated with a higher survival rate than other therapies at relapse. ${ }^{27}$ However, medulloblastoma is a radioand chemosensitive tumor, and cure may occasionally be achieved even without surgery. For a long time, it has been accepted that GTR and NTR allowing a residual tumor $\leq$ $1.5 \mathrm{~cm}^{2}$ do not differ with respect to survival outcome. The PNET 4 study confirmed this finding ${ }^{20}$ but showed that a residual tumor $>1.5 \mathrm{~cm}^{2}$, i.e., STR, implied a higher risk for relapse in the PNET 4 study ${ }^{20}$ similar to the older studies of Albright and Zeltzer.,39 No difference in 5-year PFS was seen comparing the extent of resection in this PNET 4 substudy where the great majority had no or a small residual tumor. However, a recent study on all medulloblastoma risk groups including large residuals did not show a significant benefit in overall survival associated with GTR when incorporating the molecular subgroup analyses. ${ }^{36}$ In our study, molecular subgroup analysis was performed in less than half of the patients. From this limited cohort, we could not draw meaningful conclusions on subgroups and tumor invasiveness or resection. In non-WNT/non-SHH standard-risk medulloblastomas, chromosomal signatures were found to be highly predictive for survival, while its variants "group 3" and "group 4" showed no difference."1

In individual patients, this question is important. Patients with STR may undergo second-look surgery to achieve GTR/NTR, or their treatment may be intensified to high-risk therapy, with increased risk of long-term side effects. , $^{2,9,31}$ With more knowledge of the distinct genetically defined medulloblastoma entities ${ }^{21}$ this decision may be easier to make. However, at the time of surgery, biological factors are not known and neurosurgical decisions have to be made based on tumor growth and location.

\section{Study Strengths and Limitations}

The strength of this study is the prospective collection of tumor growth pattern in a multicenter setting, which should support its universality. Also, the postoperative tumor status was centrally reviewed by MR. Although we do not have information on every type of neurological complication, we cover those most important after posterior fossa surgery.

The limitations of this study are a lack of consensus on the tumor growth definitions before the study start. The neurosurgeons may have judged this differently, and we had no information on tumor invasiveness from preoperative MRI. We cannot exclude that tumors with a cumbersome and long operation more often could be reported with invasive growth. On the other hand, judgments were made by a large group of neurosurgeons, which should limit bias. There was no information about the neurosurgical approach or the presence of pre- and postoperative hydrocephalus, albeit the need for hydrocephalus management was recorded. These factors may have had an impact on postoperative complications. Finally, the occurrence of CMS was clinically determined, without the use of a systematic scale, which could have underestimated the incidence of milder forms of CMS.

\section{Conclusions}

In this multicenter prospective study, the overall frequency of neurological impairments secondary to medulloblastoma surgery remained stable, both pre- and postoperatively, at $70 \%$. Interestingly, postoperative neurological damage was mainly due to preoperative factors, including invasive tumor growth. This affected short-term neurological outcome, whereas the extent of resection did not. This somewhat unexpected finding needs to be confirmed in other studies but may be the result of the current recommendations with maximal safe resection where functional risks are not taken in areas with tumor invasion.

\section{Acknowledgments}

The contributions to this study from the SIOP-E Brain Tumor Group, national study group members, and national data managers are gratefully acknowledged. This work was supported by the Swedish Childhood Cancer Foundation, Cancer Research UK, the French Ministry of Health, the French National Cancer Institute (INCa), the German Children's Cancer Foundation, and Associazione Bianca Garavaglia onlus (B. Arsizio, Milano).

\section{References}

1. Albright AL, Wisoff JH, Zeltzer PM, Boyett JM, Rorke LB, Stanley P: Effects of medulloblastoma resections on outcome in children: a report from the Children's Cancer Group. Neurosurgery 38:265-271, 1996

2. Armstrong GT, Liu Q, Yasui Y, Huang S, Ness KK, Leisenring W, et al: Long-term outcomes among adult survivors of childhood central nervous system malignancies in the Childhood Cancer Survivor Study. J Natl Cancer Inst 101:946-958, 2009

3. Avula S, Mallucci C, Kumar R, Pizer B: Posterior fossa syndrome following brain tumour resection: review of pathophysiology and a new hypothesis on its pathogenesis. Childs Nerv Syst 31:1859-1867, 2015 
4. Boman KK, Lindblad F, Hjern A: Long-term outcomes of childhood cancer survivors in Sweden: a population-based study of education, employment, and income. Cancer 116:1385-1391, 2010

5. Brasme JF, Grill J, Doz F, Lacour B, Valteau-Couanet D, Gaillard S, et al: Long time to diagnosis of medulloblastoma in children is not associated with decreased survival or with worse neurological outcome. PLoS One 7:e33415, 2012

6. Câmara-Costa H, Resch A, Kieffer V, Lalande C, Poggi G, Kennedy C, et al: Neuropsychological outcome of children treated for standard risk medulloblastoma in the PNET4 European randomized controlled trial of hyperfractionated versus standard radiation therapy and maintenance chemotherapy. Int J Radiat Oncol Biol Phys 92:978-985, 2015

7. Cochrane DD, Gustavsson B, Poskitt KP, Steinbok P, Kestle JR: The surgical and natural morbidity of aggressive resection for posterior fossa tumors in childhood. Pediatr Neurosurg 20:19-29, 1994

8. Doxey D, Bruce D, Sklar F, Swift D, Shapiro K: Posterior fossa syndrome: identifiable risk factors and irreversible complications. Pediatr Neurosurg 31:131-136, 1999

9. Frange P, Alapetite C, Gaboriaud G, Bours D, Zucker JM, Zerah M, et al: From childhood to adulthood: long-term outcome of medulloblastoma patients. The Institut Curie experience (1980-2000). J Neurooncol 95:271-279, 2009

10. Gajjar A, Sanford RA, Bhargava R, Heideman R, Walter A, Li Y, et al: Medulloblastoma with brain stem involvement: the impact of gross total resection on outcome. Pediatr Neurosurg 25:182-187, 1996

11. Goschzik T, Schwalbe EC, Hicks D, Smith A, Zur Muehlen A, Figarella-Branger D, et al: Prognostic effect of whole chromosomal aberration signatures in standard-risk, non$\mathrm{WNT} /$ non-SHH medulloblastoma: a retrospective, molecular analysis of the HIT-SIOP PNET 4 trial. Lancet Oncol 19:1602-1616, 2018

12. Grill J, Sainte-Rose C, Jouvet A, Gentet JC, Lejars O, Frappaz D, et al: Treatment of medulloblastoma with postoperative chemotherapy alone: an SFOP prospective trial in young children. Lancet Oncol 6:573-580, 2005

13. Grill J, Viguier D, Kieffer V, Bulteau C, Sainte-Rose C, Hartmann O, et al: Critical risk factors for intellectual impairment in children with posterior fossa tumors: the role of cerebellar damage. J Neurosurg 101 (2 Suppl):152-158, 2004

14. Gudrunardottir T, Morgan AT, Lux AL, Walker DA, Walsh KS, Wells EM, et al: Consensus paper on post-operative pediatric cerebellar mutism syndrome: the Iceland Delphi results. Childs Nerv Syst 32:1195-1203, 2016

15. Gudrunardottir T, Sehested A, Juhler M, Schmiegelow K: Cerebellar mutism: review of the literature. Childs Nerv Syst 27:355-363, 2011

16. Houdemont SP, De Carli E, Delion M, Ringuier B, Chapotte C, Jeudy C, et al: Short-term neurological outcome of children after surgery for brain tumors: incidence and characteristics in a pediatric intensive care unit. Childs Nerv Syst 27:933-941, 2011

17. Huber JF, Bradley K, Spiegler B, Dennis M: Long-term neuromotor speech deficits in survivors of childhood posterior fossa tumors: effects of tumor type, radiation, age at diagnosis, and survival years. J Child Neurol 22:848-854, 2007

18. Kennedy C, Bull K, Chevignard M, Culliford D, Dörr HG, Doz F, et al: Quality of survival and growth in children and young adults in the PNET4 European controlled trial of hyperfractionated versus conventional radiation therapy for standard-risk medulloblastoma. Int J Radiat Oncol Biol Phys 88:292-300, 2014

19. Korah MP, Esiashvili N, Mazewski CM, Hudgins RJ, Tighiouart M, Janss AJ, et al: Incidence, risks, and sequelae of posterior fossa syndrome in pediatric medulloblastoma. Int J Radiat Oncol Biol Phys 77:106-112, 2010
20. Lannering B, Rutkowski S, Doz F, Pizer B, Gustafsson G, Navajas A, et al: Hyperfractionated versus conventional radiotherapy followed by chemotherapy in standard-risk medulloblastoma: results from the randomized multicenter HIT-SIOP PNET 4 trial. J Clin Oncol 30:3187-3193, 2012

21. Louis DN, Perry A, Reifenberger G, von Deimling A, Figarella-Branger D, Cavenee WK, et al: The 2016 World Health Organization Classification of Tumors of the Central Nervous System: a summary. Acta Neuropathol 131:803-820, 2016

22. Mulhern RK, Merchant TE, Gajjar A, Reddick WE, Kun LE: Late neurocognitive sequelae in survivors of brain tumours in childhood. Lancet Oncol 5:399-408, 2004

23. Northcott PA, Robinson GW, Kratz CP, Mabbott DJ, Pomeroy SL, Clifford SC, et al: Medulloblastoma. Nat Rev Dis Primers 5:11, 2019

24. Pietsch T, Schmidt R, Remke M, Korshunov A, Hovestadt V, Jones DT, et al: Prognostic significance of clinical, histopathological, and molecular characteristics of medulloblastomas in the prospective HIT2000 multicenter clinical trial cohort. Acta Neuropathol 128:137-149, 2014

25. Pollack IF, Polinko P, Albright AL, Towbin R, Fitz C: Mutism and pseudobulbar symptoms after resection of posterior fossa tumors in children: incidence and pathophysiology. Neurosurgery 37:885-893, 1995

26. Robertson PL, Muraszko KM, Holmes EJ, Sposto R, Packer $\mathrm{RJ}$, Gajjar A, et al: Incidence and severity of postoperative cerebellar mutism syndrome in children with medulloblastoma: a prospective study by the Children's Oncology Group. J Neurosurg 105 (6 Suppl):444-451, 2006

27. Sabel M, Fleischhack G, Tippelt S, Gustafsson G, Doz F, Kortmann R, et al: Relapse patterns and outcome after relapse in standard risk medulloblastoma: a report from the HIT-SIOP-PNET4 study. J Neurooncol 129:515-524, 2016

28. Schwalbe EC, Hicks D, Rafiee G, Bashton M, Gohlke H, Enshaei A, et al: Minimal methylation classifier (MIMIC): a novel method for derivation and rapid diagnostic detection of disease-associated DNA methylation signatures. Sci Rep 7:13421, 2017

29. Schwalbe EC, Lindsey JC, Nakjang S, Crosier S, Smith AJ, Hicks D, et al: Novel molecular subgroups for clinical classification and outcome prediction in childhood medulloblastoma: a cohort study. Lancet Oncol 18:958-971, 2017

30. Schwalbe EC, Williamson D, Lindsey JC, Hamilton D, Ryan SL, Megahed H, et al: DNA methylation profiling of medulloblastoma allows robust subclassification and improved outcome prediction using formalin-fixed biopsies. Acta Neuropathol 125:359-371, 2013

31. Schwartz CL: Long-term survivors of childhood cancer: the late effects of therapy. Oncologist 4:45-54, 1999

32. Sønderkaer S, Schmiegelow M, Carstensen H, Nielsen LB, Müller J, Schmiegelow K: Long-term neurological outcome of childhood brain tumors treated by surgery only. J Clin Oncol 21:1347-1351, 2003

33. Steinbok P, Cochrane DD, Perrin R, Price A: Mutism after posterior fossa tumour resection in children: incomplete recovery on long-term follow-up. Pediatr Neurosurg 39:179_ 183,2003

34. Taylor MD, Northcott PA, Korshunov A, Remke M, Cho YJ, Clifford SC, et al: Molecular subgroups of medulloblastoma: the current consensus. Acta Neuropathol 123:465-472, 2012

35. Thompson EM, Bramall A, Herndon JE II, Taylor MD, Ramaswamy V: The clinical importance of medulloblastoma extent of resection: a systematic review. J Neurooncol 139:523-539, 2018

36. Thompson EM, Hielscher T, Bouffet E, Remke M, Luu B, Gururangan S, et al: Prognostic value of medulloblastoma extent of resection after accounting for molecular subgroup: a retrospective integrated clinical and molecular analysis. Lancet Oncol 17:484-495, 2016 
37. van Veelen-Vincent ML, Pierre-Kahn A, Kalifa C, SainteRose C, Zerah M, Thorne J, et al: Ependymoma in childhood: prognostic factors, extent of surgery, and adjuvant therapy. J Neurosurg 97:827-835, 2002

38. Wolfe KR, Madan-Swain A, Kana RK: Executive dysfunction in pediatric posterior fossa tumor survivors: a systematic literature review of neurocognitive deficits and interventions. Dev Neuropsychol 37:153-175, 2012

39. Zeltzer PM, Boyett JM, Finlay JL, Albright AL, Rorke LB, Milstein JM, et al: Metastasis stage, adjuvant treatment, and residual tumor are prognostic factors for medulloblastoma in children: conclusions from the Children's Cancer Group 921 randomized phase III study. J Clin Oncol 17:832-845, 1999

\section{Disclosures}

The authors report no conflict of interest concerning the materials or methods used in this study or the findings specified in this paper.

\section{Author Contributions}

Conception and design: Schepke, Lannering, Tisell. Analysis and interpretation of data: Schepke, Tisell, Lannering. Drafting the article: Schepke, Tisell, Kennedy, Lannering. Critically revising the article: Tisell, Kennedy, Puget, Ferroli, Chevignard, Doz, Pizer, Rutkowski, Massimino, Navajas, Schwalbe, Hicks, Clifford, Pietsch, Lannering. Reviewed submitted version of manuscript: Schepke, Tisell, Kennedy, Chevignard, Lannering. Statistical analysis: Schepke. Study supervision: Lannering. Performed methylation analyses: Schwalbe, Hicks.

\section{Supplemental Information}

\section{Previous Presentations}

Portions of this work were presented in abstract form and as an oral presentation at the 67th Scandinavian Neurosurgical Congress, Stockholm, Sweden, May 20, 2019.

\section{Correspondence}

Elizabeth Schepke: Queen Silvias Children's Hospital, Sahlgrenska University Hospital, Gothenburg, Sweden. elizabeth. schepke@vgregion.se. 